\title{
The Hubble constant determined through an inverse distance ladder including quasar time delays and Type la supernovae
}

\author{
S. Taubenberger ${ }^{1}$, S. H. Suyu ${ }^{1,2,3}$, E. Komatsu ${ }^{1,4}$, I. Jee ${ }^{1}$, S. Birrer ${ }^{5}$, V. Bonvin ${ }^{6}$, F. Courbin ${ }^{6}$, C. E. Rusu ${ }^{7,8,9}$, \\ A. J. Shajib ${ }^{5}$, and K. C. Wong ${ }^{4,7}$ \\ 1 Max-Planck-Institut für Astrophysik, Karl-Schwarzschild-Str. 1, 85748 Garching, Germany \\ e-mail: tauben@mpa-garching.mpg.de \\ 2 Physik-Department, Technische Universität München, James-Franck-Str. 1, 85748 Garching, Germany \\ 3 Institute of Astronomy and Astrophysics, Academia Sinica, 11F of ASMAB, No.1, Section 4, Roosevelt Rd., Taipei 10617, Taiwan \\ ${ }^{4}$ Kavli Institute for the Physics and Mathematics of the Universe (Kavli IPMU, WPI), Univ. of Tokyo, Kashiwa 277-8583, Japan \\ 5 Department of Physics and Astronomy, University of California, Los Angeles, CA 90095-1547, USA \\ ${ }^{6}$ Laboratoire d'Astrophysique, École Polytechnique Fédérale de Lausanne (EPFL), Observatoire de Sauverny, 1290 Versoix, \\ Switzerland \\ 7 National Astronomical Observatory of Japan, 2-21-1 Osawa, Mitaka, Tokyo 181-8588, Japan \\ 8 Subaru Telescope, National Astronomical Observatory of Japan, 650 N Aohoku Pl, Hilo 96720, Japan \\ 9 Department of Physics, University of California, Davis, 1 Shields Avenue, Davis, CA 95616, USA
}

Received 29 May 2019 / Accepted 20 July 2019

\begin{abstract}
Context. The precise determination of the present-day expansion rate of the Universe, expressed through the Hubble constant $H_{0}$, is one of the most pressing challenges in modern cosmology. Assuming flat $\Lambda \mathrm{CDM}, H_{0}$ inference at high redshift using cosmic microwave background data from Planck disagrees at the $4.4 \sigma$ level with measurements based on the local distance ladder made up of parallaxes, Cepheids, and Type Ia supernovae (SNe Ia), often referred to as Hubble tension. Independent cosmological-modelinsensitive ways to infer $H_{0}$ are of critical importance.

Aims. We apply an inverse distance ladder approach, combining strong-lensing time-delay distance measurements with SN Ia data. By themselves, SNe Ia are merely good indicators of relative distance, but by anchoring them to strong gravitational lenses we can obtain an $H_{0}$ measurement that is relatively insensitive to other cosmological parameters.

Methods. A cosmological parameter estimate was performed for different cosmological background models, both for strong-lensing data alone and for the combined lensing $+\mathrm{SNe}$ Ia data sets.

Results. The cosmological-model dependence of strong-lensing $H_{0}$ measurements is significantly mitigated through the inverse distance ladder. In combination with SN Ia data, the inferred $H_{0}$ consistently lies around $73-74 \mathrm{~km} \mathrm{~s}^{-1} \mathrm{Mpc}^{-1}$, regardless of the assumed cosmological background model. Our results agree closely with those from the local distance ladder, but there is a $>2 \sigma$ tension with Planck results, and a $\sim 1.5 \sigma$ discrepancy with results from an inverse distance ladder including Planck, baryon acoustic oscillations, and SNe Ia. Future strong-lensing distance measurements will reduce the uncertainties in $H_{0}$ from our inverse distance ladder.
\end{abstract}

Key words. gravitational lensing: strong - cosmological parameters - distance scale

\section{Introduction}

Ever since Georges Lemaître and Edwin Hubble discovered that our Universe is expanding (Lemaître 1927, 1931; Hubble 1929), astronomers have sought to measure the Hubble constant $H_{0}$ that characterises the present-day expansion rate. For decades there was the "factor of 2 controversy" in the $H_{0}$ measurements, culminating in the Hubble Space Telescope Key Project that pinned down $H_{0}$ to $72 \pm 8 \mathrm{~km} \mathrm{~s}^{-1} \mathrm{Mpc}^{-1}$ using the Cepheids distance ladder with several secondary distance indicators including Type Ia Supernovae (SNe Ia) (Freedman et al. 2001; Freedman 2017). In recent years, another controversy on $H_{0}$ has emerged, particularly between the measurements based on the cosmic microwave background $\left(\mathrm{CMB} ; H_{0}=67.36 \pm 0.54 \mathrm{~km} \mathrm{~s}^{-1} \mathrm{Mpc}^{-1}\right.$ for flat $\Lambda C D M$; Planck Collaboration VI 2018) and the local distance ladder (SHOES programme; $H_{0}=74.03 \pm 1.42 \mathrm{~km} \mathrm{~s}^{-1} \mathrm{Mpc}^{-1}$; Riess et al. 2019). The value of $H_{0}$ inferred from the CMB depends on the background cosmology, and the $4.4 \sigma$ tension between the Planck and SHOES measurements refers to a standard flat $\Lambda \mathrm{CDM}$ cosmology with a spatially flat Universe consisting of cold dark matter and a dark energy that is described by the cosmological constant $\Lambda$.

This tension, if not resolved by systematic effects (e.g., Rigault et al. 2015, 2018; Jones et al. 2018; Roman et al. 2018), indicates new physics beyond flat $\Lambda$ CDM (e.g., Di Valentino et al. 2018; Mörtsell \& Dhawan 2018; Adhikari \& Huterer 2019; Agrawal et al. 2019; Kreisch et al. 2019; Pandey et al. 2019; Poulin et al. 2019; Vattis et al. 2019). Independent measurements of $H_{0}$ are particularly important in order to verify this tension, given the potential of discovering new physics. Methods based on Type IIP supernova expanding photospheres (Schmidt et al. 1994; Gall et al. 2016), water masers (Gaoet al.2016; Braatzet al.2019), or standard sirens (Schutz 1986; The LIGO Scientific Collaboration et al. 2017) have recently provided independent $H_{0}$ measurements. While they currently have uncertainties that are consistent with both the 
Planck and the SH0ES measurements, future measurements with larger samples of Type IIP supernovae, water masers, and standard sirens could reduce their uncertainties to help shed light on the $H_{0}$ tension.

Gravitationally lensed quasars are another competitive and independent cosmological probe, particularly in measuring $H_{0}$ When a quasar is strongly lensed by a foreground galaxy, multiple time-delayed images of the quasar appear around the lens. By measuring the time delays between the multiple quasar images and modelling the mass distributions of both the lens galaxy and other structures along the line of sight, strong lensing provides a measurement of the time-delay distance $\left(D_{\Delta t}\right)$, which is a combination of the angular diameter distances between the observer and the lens $\left(D_{\mathrm{d}}\right)$, the observer and the quasar $\left(D_{\mathrm{s}}\right)$, and the lens and the quasar $\left(D_{\mathrm{ds}}\right): D_{\Delta t}=\left(1+z_{\mathrm{d}}\right) D_{\mathrm{d}} D_{\mathrm{s}} / D_{\mathrm{ds}}(\operatorname{Refsdal} 1964$; Suyu et al. 2010; Treu \& Marshall 2016). While $D_{\Delta t}$ is inversely proportional and mostly (but not only) sensitive to $H_{0}$, the inference of $H_{0}$ from $D_{\Delta t}$ measurements depends on the cosmological model. In addition, stellar velocity dispersions of the foreground lens galaxies, if available, provide a determination of $D_{\mathrm{d}}$, which can further constrain cosmological models (Paraficz \& Hjorth 2009; Jee et al. 2015, 2016, 2019).

The HOLiCOW project (Suyu et al. 2017), in Collaboration with the COSMOGRAIL programme (Courbin et al. 2018), has assembled a sample of lensed quasar systems with exquisitely measured time-delay distances (Bonvin et al. 2017; Wong et al. 2017; Birrer et al. 2019). Through a blind analysis, Birrer et al. (2019) reported $H_{0}=72.5_{-2.3}^{+2.1} \mathrm{~km} \mathrm{~s}^{-1} \mathrm{Mpc}^{-1}$ (3\% uncertainty, including systematics) from the data of four lensed quasars, in flat $\Lambda$ CDM. However, as in all cosmological experiments that measure distances outside the scope of the linear Hubble relation $D=c z / H_{0}$, the inference of $H_{0}$ from $D_{\Delta t}$ depends on the assumed background cosmology. While much focus in the community is on $H_{0}$ in flat $\Lambda \mathrm{CDM}$, a cosmological-modelindependent inference of $H_{0}$ is valuable.

The inverse distance ladder (Aubourg et al. 2015; Cuesta et al. 2015) provides a way to infer $H_{0}$ which is more modelindependent. The idea is to anchor the relative distances from SNe Ia with an absolute distance measurement. Supernova distances on their own are not absolute distances because of the unknown intrinsic luminosity of $\mathrm{SNe}$ (e.g. Leibundgut et al. 2017). Nonetheless, SNe map out the expansion history of the Universe very precisely, and by anchoring their distance scale with absolute distance measurements, cosmological-modelinsensitive absolute distance determinations become feasible. By anchoring the SN distance scale using distances measured from baryon acoustic oscillations (BAO), Macaulay et al. (2019) measured an $H_{0}$ from the Dark Energy Survey consistent with that provided by the Planck Collaboration VI (2018) and that does not depend much on cosmological models, although the inference of $H_{0}$ is strongly affected by the assumptions of the size of the sound horizon (Aylor et al. 2019). Recently, Jee et al. (2019) and Wojtak \& Agnello (2019) anchored the SN distance scale using $D_{\mathrm{d}}$ measured from strongly lensed quasars, resulting in $H_{0}$ values with $\sim 10 \%$ uncertainty, limited by the precision of the $D_{\mathrm{d}}$ measurements. With current data, lensed quasars yield tighter constraints on $D_{\Delta t}$ than $D_{\mathrm{d}}$. In this paper, we explore the use of $D_{\Delta t}$ as anchor.

This Letter is organised as follows. In Sect. 2 we summarise the $D_{\Delta t}$ measurements from the four HOLiCOW lenses, and in Sect. 3 we use these distances to infer $H_{0}$ through the inverse distance ladder. We discuss the results in Sect. 4, and provide an outlook in Sect. 5. Throughout the paper, our parameter constraints correspond to the median values of the parameter
Table 1. Lens redshifts $\left(z_{\mathrm{d}}\right)$ and source redshifts $\left(z_{\mathrm{s}}\right)$ of the strongly lensed quasars from HOLiCOW included in this study.

\begin{tabular}{llll}
\hline \hline Name & $z_{\mathrm{d}}$ & $z_{\mathrm{s}}$ & References \\
\hline B1608+656 & 0.6304 & 1.394 & 1,2 \\
RX J1131-1231 & 0.295 & 0.654 & 3,2 \\
HE 0435-1223 & 0.4546 & 1.693 & 4,2 \\
SDSS 1206+4332 & 0.745 & 1.789 & 5 \\
\hline
\end{tabular}

References. (1) Suyu et al. (2010); (2) Bonvin et al. (2017); (3) Suyu et al. (2014); (4) Wong et al. (2017); (5) Birrer et al. (2019)

probability distributions, with $68 \%$ credibility intervals given by the 16 th and 84 th percentiles.

\section{Lensing time-delay distances}

We use the posterior probability distribution of $D_{\Delta t}, P\left(D_{\Delta t}\right)$, for the four lensed quasar systems that have been measured by the HOLiCOW Collaboration (listed in Table 1). For three systems (B1608+656, RX J1131-1231, and HE 0435-1223; Suyu et al. 2010, 2014; Sluse et al. 2017; Rusu et al. 2017; Wong et al. 2017; Tihhonova et al. 2018), $P\left(D_{\Delta t}\right)$ is nicely described by the analytic fit

$P\left(D_{\Delta t}\right)=\frac{1}{\sqrt{2 \pi}\left(x-\lambda_{\mathrm{D}}\right) \sigma_{\mathrm{D}}} \exp \left[-\frac{\left(\ln \left(x-\lambda_{\mathrm{D}}\right)-\mu_{\mathrm{D}}\right)^{2}}{2 \sigma_{\mathrm{D}}^{2}}\right]$,

where $x=D_{\Delta t} /(1 \mathrm{Mpc})$, and the fitted parameter values $\left(\lambda_{\mathrm{D}}\right.$, $\left.\sigma_{\mathrm{D}}, \mu_{\mathrm{D}}\right)$ are listed in Table 3 of Bonvin et al. (2017). For the fourth lens system (SDSS 1206+4332), we use the Markov chain Monte Carlo (MCMC) results for $D_{\Delta t}$ from Birrer et al. (2019) ${ }^{1}$, and obtain $P\left(D_{\Delta t}\right)$ through a kernel density estimator.

\section{Inverse distance ladder: supernovae anchored with strongly lensed quasars}

To determine the joint likelihood of cosmological parameters for different experiments and cosmological models, we employ the MontePython v3.1 MCMC sampling package (Audren et al. 2013; Brinckmann \& Lesgourgues 2018), which is interfaced with the Boltzmann code CLASS (Lesgourgues 2011) for CMB calculations. As MCMC algorithm, we select MontePython's Metropolis-Hastings sampler. For every combination of cosmological probes and assumed cosmological background model, we start with a relatively short MCMC chain ( 50000 steps) with dynamic updates of the covariance matrix and jumping factor (known as the super-update strategy in MontePython; Brinckmann \& Lesgourgues 2018), which ensures an efficient sampling and an acceptance rate close to the optimal $25 \%$. This is followed by a fully Markovian chain of 500000 steps, where the covariance matrix and jumping factor are kept fixed at the previously determined values. Our long chains have acceptance rates between $15 \%$ and $30 \%$ and show good convergence.

The sampling includes the $H_{0}$ and $\Omega_{\mathrm{CDM}}$ parameters ${ }^{2}$ and, for cosmological models other than flat $\Lambda \mathrm{CDM}$, combinations of $\Omega_{k}, w_{0}$, and $w_{a}$. The priors employed for these cosmological parameters are summarised in Table 2 . They can have an impact

1 The chain of the joint constraint on $D_{\Delta t}$ and $D_{\mathrm{d}}$ is released on the HOLiCOW website (http://wwW.holicow.org), and we focus on $D_{\Delta t}$, marginalising over $D_{\mathrm{d}}$.

2 The baryon energy density $\Omega_{\mathrm{b}}$ is fixed at 0.05 , so that $\Omega_{\mathrm{m}}$ and $\Omega_{\mathrm{CDM}}$ can be used interchangeably. 
Table 2. Priors on cosmological parameters (all uniform) as employed in the MontePython MCMC sampling.

\begin{tabular}{lcc}
\hline \hline Parameter & Minimum & Maximum \\
\hline$\Omega_{\mathrm{CDM}}$ & 0 & 0.45 \\
$\Omega_{k}$ & -0.2 & 0.2 \\
$w_{0}$ & -2.5 & 0.5 \\
$w_{a}$ & -2.0 & 2.0 \\
$H_{0}$ & 0 & 150 \\
\hline
\end{tabular}

on the inferred parameter posteriors, so we make sure that they are either physically motivated or sufficiently conservative. In those runs where strong-lensing data are combined with SN Ia data, four additional nuisance parameters $\left(M_{B}, \alpha, \beta\right.$, and $\left.\Delta_{M}\right)$ are added. They represent the absolute $B$-band magnitude, the coefficients of light curve stretch $\left(X_{1}\right)$ and colour $(C)$ corrections, and the host-galaxy mass step, respectively, in a SALT2 framework (Guy et al. 2007; Mosher et al. 2014; Betoule et al. 2014):

$\mu=m_{B}-\left(M_{B}-\alpha \times X_{1}+\beta \times C+\Delta_{M}\right)$.

The (luminosity) distance modulus, $\mu=5 \log _{10}\left(D_{\mathrm{L}} / 1 \mathrm{Mpc}\right)+$ 25 , is thereby calculated as the difference between the apparent peak magnitude in the rest frame $B$ band $\left(m_{B}\right)$, and the stretchand colour-corrected absolute $B$-band magnitude. The empirical mass step-correction $\Delta_{M}$ is only added for $\mathrm{SN}$ host galaxies with stellar masses $\geq 10^{10} M_{\odot}$ (Sullivan et al. 2010).

We first concentrate on the cosmological parameter inference using HOLiCOW $D_{\Delta t}$ data of strongly lensed quasars alone. Four different background cosmologies are investigated: flat $\Lambda \mathrm{CDM}$; flat $w C D M$ with a redshift-independent dark energy equationof-state parameter $w$, which can differ from -1 (corresponding to $\Lambda$ ); flat $w_{0} w_{a} \mathrm{CDM}$ with a redshift-dependent dark energy equation-of-state parameter $w(z)=w_{0}+w_{a} \frac{z}{1+z}$; and non-flat $\Lambda \mathrm{CDM}$, which covers the possibilities of a negatively or positively curved Universe. The resulting cosmological parameters are shown in Table 3 . The energy densities of matter $\left(\Omega_{\mathrm{m}}\right)$, a cosmological constant $\left(\Omega_{\Lambda}\right)$, or a more generic form of dark energy $\left(\Omega_{\mathrm{DE}}\right)$ are not tightly constrained by the lensed quasars alone, but the effect of different background cosmologies is very weak for these parameters. For non- $\Lambda \mathrm{CDM}$ models, $w$ deviates from -1 by more than $1 \sigma$, while the curvature in the non-flat $\Lambda \mathrm{CDM}$ case is consistent with zero. The Hubble constant shows a moderately strong dependence on the background cosmology, in particular on the dark energy equation of state, being 72.9 in both the flat and non-flat $\Lambda \mathrm{CDM}$ cases, but $>80$ in the flat $w C D M$ and $w_{0} w_{a} \mathrm{CDM}$ cosmologies. This can be explained by the lensedquasar systems spanning redshifts between $\sim 0.3$ (for the most nearby lens) and $\sim 1.8$ (for the most distant quasar; see Table 1), and the necessary extrapolation to obtain the present-day expansion rate of the Universe being cosmological-model-dependent, for example due to degeneracy between $H_{0}$ and $w$ (Fig. 1).

The dependence of $H_{0}$ on the assumed background cosmology can be mitigated by combining the quasar $D_{\Delta t}$ measurements with SN data from the joint light curve analysis (JLA), which is a compilation of 740 spectroscopically confirmed low- $z$, SDSSII, and SNLS SNe Ia (Betoule et al. 2014). In this inverse distance ladder approach, the SN Ia data are anchored near their high- $z$ end by the lensed quasars, and allow for a much improved measurement of the present-day expansion rate compared to the quasar time delays alone. We employ the same priors on cosmological parameters as before (Table 2). In addition to the cosmological models investigated in the lenses-only case, we now also include the more flexible non-flat $w \mathrm{CDM}$ and non-flat $w_{0} w_{a} \mathrm{CDM}$ models, which did not converge in the lenses-only MCMC chains. The results are again summarised in Table 3. The posteriors for $\Omega_{\mathrm{m}}$ and $\Omega_{\Lambda}$ (or $\Omega_{\mathrm{DE}}$ ) have tightened up significantly compared to the lenses-only case, which is a merit of SNe Ia being able to map out the relative expansion history of the Universe very well. Similarly, the dark energy equation-of-state parameter $w$ in the non- $\Lambda \mathrm{CDM}$ models is now better constrained, and very close to -1 in all models. The inferred curvature in the non-flat geometries is slightly larger than before, but still consistent with zero. The Hubble constant, finally, shows only a $2 \%$ variation with the assumed background cosmology, lying between 72.7 and $74.1 \mathrm{~km} \mathrm{~s}^{-1} \mathrm{Mpc}^{-1}$ in all cases. The increased median and uncertainty observed for non- $\Lambda$ CDM cosmologies obtained from lensed quasars alone is no longer an issue when combined with SN Ia data.

For two selected background cosmologies (flat $w \mathrm{CDM}$ and non-flat $\Lambda \mathrm{CDM}$ ), the full posterior distributions for the sampled cosmological parameters are shown in Fig. 1. The improved constraints on $H_{0}$ and $w$ (in flat $w \mathrm{CDM}$ ) and on $\Omega_{\mathrm{m}}$ (in non-flat $\Lambda \mathrm{CDM}$ ) when including SN Ia data are evident. Figure 2 compares the marginalised $1 \mathrm{D} H_{0}$ posteriors obtained from lensed quasars alone with those obtained from a combination of lensing and SN Ia data for different cosmological models. Using the inverse distance ladder, the peak-to-peak scatter in the median $H_{0}$ values of these four models is impressively reduced from $11 \%$ to $1 \%$.

\section{Discussion}

We now investigate how our $H_{0}$ results compare to those from other cosmological probes: the SH0ES (Riess et al. 2019) and Planck (Planck Collaboration VI 2018) experiments, and a Planck $+\mathrm{BAO}+$ SNe Ia inverse distance ladder (Aubourg et al. 2015).

The local distance ladder underlying the SHOES determination of $H_{0}$ is anchored to parallaxes at $z=0$. It is therefore almost completely insensitive to the cosmological background model. As shown in Fig. 3, our inverse distance ladder measurements of $H_{0}$ for different cosmologies all agree very nicely with the SH0ES results of $H_{0}=74.03 \pm 1.42 \mathrm{~km} \mathrm{~s}^{-1} \mathrm{Mpc}^{-1}$.

Inference of $H_{0}$ based on $\mathrm{CMB}$, on the contrary, takes place at $z>1000$, and involves extrapolation to $z=0$ by assuming a cosmological model. Hence, while the Planck results for $H_{0}$ are very precise once a flat $\Lambda \mathrm{CDM}$ cosmology is assumed, they can vary widely if this assumption is dropped. The Planck Collaboration VI (2018) provides $H_{0}$ values only for flat $\Lambda \mathrm{CDM}$ $\left(H_{0}=67.36 \pm 0.54 \mathrm{~km} \mathrm{~s}^{-1} \mathrm{Mpc}^{-1}\right)$ and non-flat $\Lambda \mathrm{CDM}\left(H_{0}=\right.$ $\left.63.6_{-2.3}^{+2.1} \mathrm{~km} \mathrm{~s}^{-1} \mathrm{Mpc}^{-1}\right)$ cosmologies, and already these differ significantly (see Fig. 3). With cosmologies that do not assume a cosmological constant, no meaningful constraints on $H_{0}$ can be obtained from the CMB alone. Our inverse distance ladder results show a tension of just above $2 \sigma$ with Planck for both flat $\Lambda C D M$ and non-flat $\Lambda \mathrm{CDM}$, which is lower than the tension between Planck and SHOES owing to our larger error bars compared to SHOES.

Finally, the Planck $+\mathrm{BAO}+\mathrm{SNe}$ Ia inverse distance ladder of Aubourg et al. (2015) is anchored to BAO absolute distances at redshifts $0.1-0.6$. CMB data are used to set the sound-horizon scale, which BAO distances are inversely proportional to. The inferred values of $H_{0}$ for different cosmological models are in good agreement with each other, clustering between 67 and $68 \mathrm{~km} \mathrm{~s}^{-1} \mathrm{Mpc}^{-1}$, with uncertainties between $1.0 \%$ and $1.5 \%$. The discrepancy with our lensing-based inverse distance ladder is between 1.3 and $1.9 \sigma$, which is not huge, but systematic. A possible origin of this discrepancy could be the adopted soundhorizon scale from Planck, which is the only early-Universe property that enters into the Aubourg et al. (2015) measurement. 
Table 3. Cosmological parameters extracted with MontePython MCMC sampling.

\begin{tabular}{lcccccccc}
\hline \hline Cosmological model & $\Omega_{\mathrm{m}}$ & $\Omega_{\mathrm{CDM}}$ & $\Omega_{\Lambda}$ & $\Omega_{\mathrm{DE}}$ & $\Omega_{\mathrm{k}}$ & $w_{0}$ & $w_{a}$ & $H_{0}$ \\
\hline Lenses only & & & & & & & & \\
Flat $\Lambda \mathrm{CDM}$ & $0.26_{-0.14}^{+0.15}$ & $0.21_{-0.14}^{+0.15}$ & $0.74_{-0.15}^{+0.14}$ & $\equiv 0$ & $\equiv 0$ & $\equiv-1$ & $\equiv 0$ & $72.9_{-2.3}^{+2.1}$ \\
Flat $w \mathrm{CDM}$ & $0.26_{-0.14}^{+0.14}$ & $0.21_{-0.14}^{+0.14}$ & $\equiv 0$ & $0.74_{-0.14}^{+0.14}$ & $\equiv 0$ & $-1.74_{-0.45}^{+0.60}$ & $\equiv 0$ & $80.8_{-7.1}^{+5.3}$ \\
Flat $w_{0} w_{a} \mathrm{CDM}$ & $0.27_{-0.14}^{+0.14}$ & $0.22_{-0.14}^{+0.14}$ & $\equiv 0$ & $0.73_{-0.14}^{+0.14}$ & $\equiv 0$ & $-1.76_{-0.44}^{+0.54}$ & $-0.26_{-1.21}^{+1.37}$ & $81.2_{-6.3}^{+5.3}$ \\
Non-flat $\Lambda \mathrm{CDM}$ & $0.26_{-0.14}^{+0.15}$ & $0.21_{-0.14}^{+0.15}$ & $0.72_{-0.18}^{+0.16}$ & $\equiv 0$ & $0.03_{-0.15}^{+0.12}$ & $\equiv-1$ & $\equiv 0$ & $72.9_{-2.4}^{+2.3}$ \\
\hline Lenses + SNe Ia & & & & & & & & \\
Flat $\Lambda \mathrm{CDM}$ & $0.30_{-0.03}^{+0.04}$ & $0.25_{-0.03}^{+0.04}$ & $0.70_{-0.04}^{+0.03}$ & $\equiv 0$ & $\equiv 0$ & $\equiv-1$ & $\equiv 0$ & $73.1_{-2.2}^{+2.1}$ \\
Flat $w \mathrm{CDM}$ & $0.28_{-0.10}^{+0.11}$ & $0.23_{-0.10}^{+0.11}$ & $\equiv 0$ & $0.72_{-0.10}^{+0.11}$ & $\equiv 0$ & $-0.96_{-0.28}^{+0.21}$ & $\equiv 0$ & $72.7_{-2.9}^{+3.0}$ \\
Flat $w_{0} w_{a} \mathrm{CDM}$ & $0.32_{-0.11}^{+0.08}$ & $0.27_{-0.11}^{+0.08}$ & $\equiv 0$ & $0.68_{-0.08}^{+0.11}$ & $\equiv 0$ & $-0.97_{-0.29}^{+0.20}$ & $-0.38_{-1.08}^{+1.01}$ & $73.1_{-3.0}^{+3.0}$ \\
Non-flat $\Lambda \mathrm{CDM}$ & $0.27_{-0.05}^{+0.06}$ & $0.22_{-0.05}^{+0.06}$ & $0.66_{-0.06}^{+0.08}$ & $\equiv 0$ & $0.08_{-0.13}^{+0.09}$ & $\equiv-1$ & $\equiv 0$ & $73.4_{-2.3}^{+2.2}$ \\
Non-flat $w \mathrm{CDM}$ & $0.27_{-0.11}^{+0.09}$ & $0.22_{-0.11}^{+0.09}$ & $\equiv 0$ & $0.65_{-0.12}^{+0.15}$ & $0.08_{-0.14}^{+0.09}$ & $-1.02_{-0.34}^{+0.24}$ & $\equiv 0$ & $73.6_{-3.3}^{+3.3}$ \\
Non-flat $w_{0} w_{a} \mathrm{CDM}$ & $0.30_{-0.10}^{+0.08}$ & $0.25_{-0.10}^{+0.08}$ & $\equiv 0$ & $0.62_{-0.11}^{+0.14}$ & $0.09_{-0.14}^{+0.08}$ & $-1.05_{-0.36}^{+0.25}$ & $-0.29_{-1.14}^{+1.02}$ & $74.1_{-3.3}^{+3.1}$ \\
\hline
\end{tabular}

Notes. Results for different cosmological models are shown for quasar time delays alone (upper four lines) and for the combination of the timedelay measurements with the JLA SN Ia sample (lower six lines). The quoted numbers are the median values, while the uncertainties correspond to the 16th and 84th percentiles.

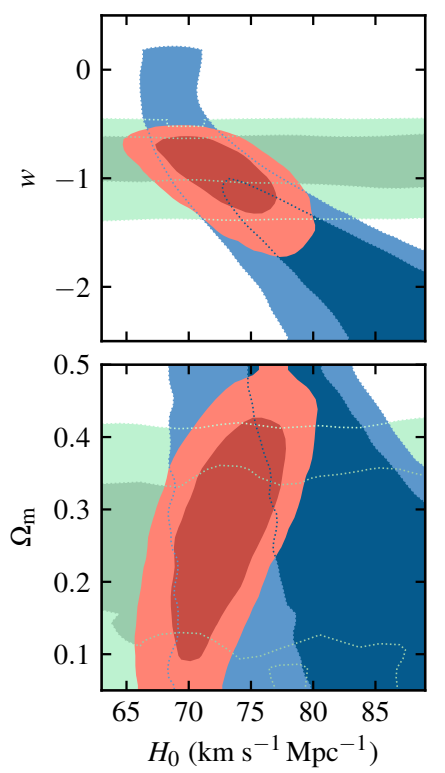

\section{flat $w \mathrm{CDM}$ \\ lenses only \\ lenses + SNe Ia \\ SNe Ia only}

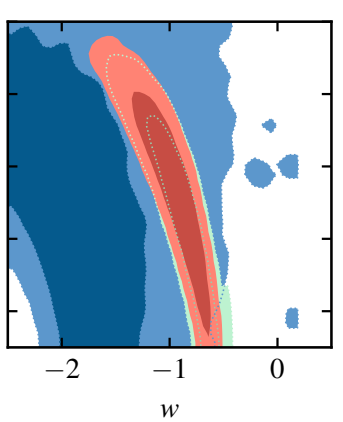

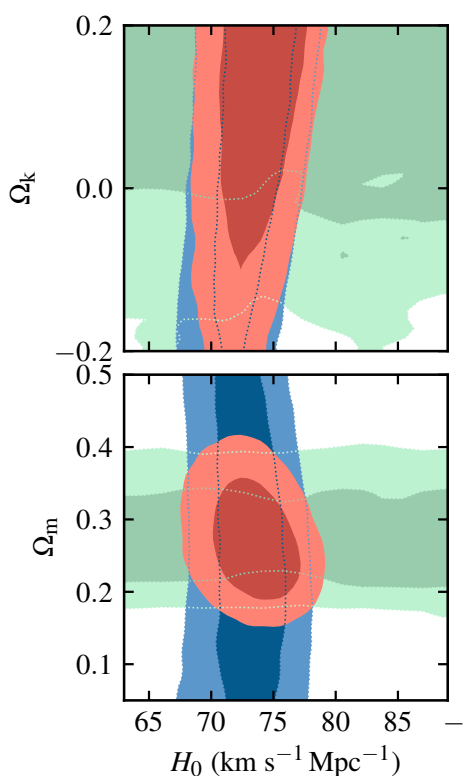

non-flat $\Lambda \mathrm{CDM}$

lenses only

lenses + SNe Ia

SNe Ia only

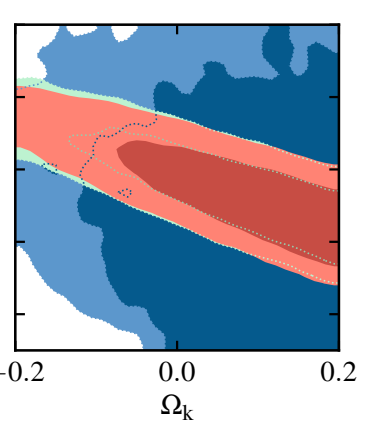

Fig. 1. Contour plots with $68 \%$ and $95 \%$ confidence regions for $H_{0}, \Omega_{\mathrm{m}}$, and $w$ in a flat $w \mathrm{CDM}$ cosmology (left-hand side), and for $H_{0}, \Omega_{\mathrm{m}}$, and $\Omega_{\mathrm{k}}$ in a non-flat $\Lambda \mathrm{CDM}$ cosmology (right-hand side). Contours based on quasar time delays and SNe Ia (JLA compilation) alone are shown in blue and green, respectively, while those using the inverse distance ladder with both probes combined are overplotted in red.

\section{Outlook}

On their own SNe Ia are poor probes of the absolute distance scale of the Universe (and hence $H_{0}$ ). In our inverse distance ladder experiment, where an anchor is provided at high redshift by time-delay distances of strongly lensed quasars, their main role is to extrapolate these absolute distance measurements back to redshift zero. This allows us to constrain $H_{0}$ in a way which is (1) rather insensitive to the assumed cosmological background model and (2) independent of Cepheids and the CMB. The Hubble constant derived from this approach (72.7-74.1 $\mathrm{km} \mathrm{s}^{-1} \mathrm{Mpc}^{-1}$ ) is consistent with that obtained with the local distance ladder, but deviates at the $\sim 1.5-2 \sigma$ level from results based on Planck CMB measurements. The origin of this discrepancy can only be speculated about, but the sound horizon determined by Planck certainly warrants further investigation.

The small $(\sim 2 \%)$ dependence of $H_{0}$ on the assumed cosmological model in our inverse distance ladder implies that the precision of the $H_{0}$ inference of $3 \%-4 \%$ is currently limited by the
$D_{\Delta t}$ data for lensed quasars. So far the number of $D_{\Delta t}$ measurements is small: merely four strongly lensed quasars have been published by the HOLiCOW Collaboration thus far. However, more are soon to come (Rusu et al. 2019a; Chen et al. 2019), and systematic searches through various surveys ${ }^{3}$ are yielding new lensed quasar systems. Some of these are now being monitored by the COSMOGRAIL Collaboration for time-delay measurements (Courbin et al. 2018; Millon et al. in prep.). With the upcoming Large Synoptic Survey Telescope (LSST) and Euclid surveys, many more $D_{\Delta t}$ measurements are expected, both for strongly lensed quasars and for $\mathrm{SNe}$. Accordingly, the statistical

3 These surveys include the Dark Energy Survey (DES) (particularly STRIDES; Treu et al. 2018), Gaia, the Hyper-Suprime Cam (HSC) survey, the Kilo-Degree Survey (KiDS), the Panoramic Survey Telescope and Rapid Response System (Pan-STARRS) and the Asteroid Terrestrial-impact Last Alert System (VST-ATLAS) (e.g., Agnello et al. 2018; Krone-Martins et al. 2018; Lemon et al. 2018; Spiniello et al. 2018; Rusu et al. 2019b). 


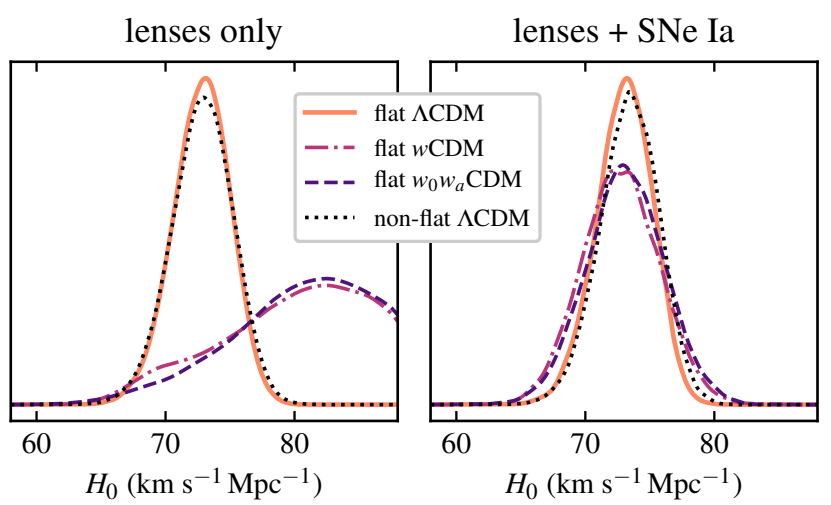

Fig. 2. $H_{0}$ posteriors for different cosmologies using HOLiCOW timedelay distance measurements of four strongly lensed quasars only (left), and using the combination of the lensing measurements with the JLA SN Ia data set (right).

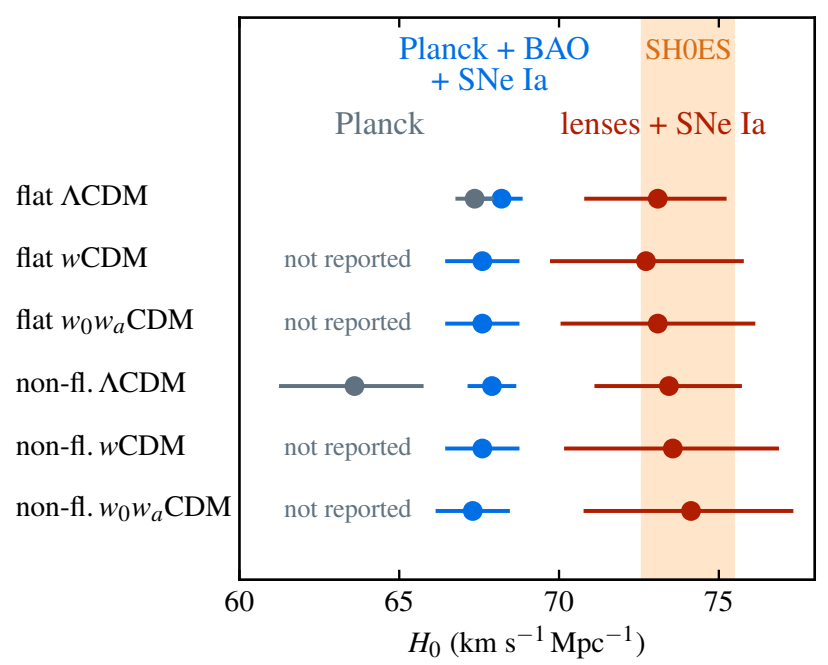

Fig. 3. Comparison between the quasar time-delay $+\mathrm{SNe}$ Ia inverse distance ladder with other cosmological probes: $\mathrm{CMB}$ data from Planck (Planck Collaboration VI 2018; TT,TE,EE + lowE + lensing), a Planck $+\mathrm{BAO}+\mathrm{SNe}$ Ia inverse distance ladder from Aubourg et al (2015), and Cepheid + SN Ia data from the SHOES project (Riess et al. 2019). The mean and $68 \%$ confidence intervals for $H_{0}$ for different background cosmologies are shown for Planck and the two inverse distance ladders. The orange-shaded region reflects the $68 \%$ confidence interval for the SHOES distance ladder, which is anchored locally and is thus insensitive to the cosmological background model.

uncertainty on $H_{0}$ from $D_{\Delta t}$ measurements will shrink substantially in the upcoming years (Shajib et al. 2018), rendering the inverse distance ladder approach that couples time-delay distances with SN Ia data one of the most promising methods for solving the current Hubble-tension puzzle.

Acknowledgements. We thank the HOLiCOW team for the public release of $D_{\Delta t}$ likelihoods. SHS thanks the Max Planck Society for the support through the Max Planck Research Group. VB and FC acknowledge support from the Swiss National Science Foundation (SNSF). This project has received funding from the European Research Council (ERC) under the EU's Horizon 2020 research and innovation programme (grant agreements No. 771776 and No. 787866). We thank Thejs Brinckmann for the helpful hints on how to run MontePython, Andreas Weiss for computing support, and the anonymous referee for constructive comments.

\section{References}

Adhikari, S., \& Huterer, D. 2019, ArXiv e-prints [arXiv:1905 . 02278] Agnello, A., Schechter, P. L., Morgan, N. D., et al. 2018, MNRAS, 475, 2086
Agrawal, P., Cyr-Racine, F. Y., Pinner, D., \& Randall, L. 2019, ArXiv e-prints [arXiv:1904.01016]

Aubourg, É., Bailey, S., Bautista, J. E., et al. 2015, Phys. Rev. D, 92, 123516

Audren, B., Lesgourgues, J., Benabed, K., \& Prunet, S. 2013, JCAP, 2, 001

Aylor, K., Joy, M., Knox, L., et al. 2019, ApJ, 874, 4

Betoule, M., Kessler, R., Guy, J., et al. 2014, A\&A, 568, A22

Birrer, S., Treu, T., Rusu, C. E., et al. 2019, MNRAS, 484, 4726

Bonvin, V., Courbin, F., Suyu, S. H., et al. 2017, MNRAS, 465, 4914

Braatz, J., Pesce, D., Condon, J., \& Reid, M. 2019, in Science with a Next-Generation VLA, ed. E. J. Murphy (San Francisco, CA: ASP), ASP Monogaph Ser. in press

Brinckmann, T., \& Lesgourgues, J. 2018, ArXiv e-prints [arXiv:1804.07261]

Chen, G. C.-F., Fassnacht, C. D., Suyu, S. H., et al. 2019, MNRAS, submitted [arXiv:1907.02533]

Courbin, F., Bonvin, V., Buckley-Geer, E., et al. 2018, A\&A, 609, A71

Cuesta, A. J., Verde, L., Riess, A., \& Jimenez, R. 2015, MNRAS, 448, 3463

Di Valentino, E., Linder, E. V., \& Melchiorri, A. 2018, Phys. Rev. D, 97, 043528

Freedman, W. L. 2017, Nat. Astron., 1, 0121

Freedman, W. L., Madore, B. F., Gibson, B. K., et al. 2001, ApJ, 553, 47

Gall, E. E. E., Kotak, R., Leibundgut, B., et al. 2016, A\&A, 592, A129

Gao, F., Braatz, J. A., Reid, M. J., et al. 2016, ApJ, 817, 128

Guy, J., Astier, P., Baumont, S., et al. 2007, A\&A, 466, 11

Hubble, E. 1929, Proc. Natl. Acad. Sci., 15, 168

Jee, I., Komatsu, E., \& Suyu, S. H. 2015, JCAP, 2015, 033

Jee, I., Komatsu, E., Suyu, S. H., \& Huterer, D. 2016, JCAP, 4, 031

Jee, I., Suyu, S. H., Komatsu, E., et al. 2019, Science, in press

Jones, D. O., Riess, A. G., Scolnic, D. M., et al. 2018, ApJ, 867, 108

Kreisch, C. D., Cyr-Racine, F. Y., \& Doré, O. 2019, ArXiv e-prints [arXiv:1902.00534]

Krone-Martins, A., Delchambre, L., Wertz, O., et al. 2018, A\&A, 616, L11

Leibundgut, B. 2017, in History of Supernovae as Distance Indicators, eds. A. W. Alsabti, \& P. Murdin (Berlin: Springer), 2525

Lemaître, G. 1927, Annales de la Société Scientifique de Bruxelles, 47, 49

Lemaître, G. 1931, MNRAS, 91, 483

Lemon, C. A., Auger, M. W., McMahon, R. G., \& Ostrovski, F. 2018, MNRAS, 479, 5060

Lesgourgues, J. 2011, ArXiv e-prints [arXiv:1104 . 2932]

Macaulay, E., Nichol, R. C., Bacon, D., et al. 2019, MNRAS, 486, 2184

Mörtsell, E., \& Dhawan, S. 2018, JCAP, 9, 025

Mosher, J., Guy, J., Kessler, R., et al. 2014, ApJ, 793, 16

Pandey, K. L., Karwal, T., \& Das, S. 2019, ArXiv e-prints [arXiv:1902 . 10636]

Paraficz, D., \& Hjorth, J. 2009, A\&A, 507, L49

Planck Collaboration VI. 2018, A\&A, submitted [arXiv:1807.06209]

Poulin, V., Smith, T. L., Karwal, T., \& Kamionkowski, M. 2019, Phys. Rev. Lett., 122,221301

Refsdal, S. 1964, MNRAS, 128, 307

Riess, A. G., Casertano, S., Yuan, W., Macri, L. M., \& Scolnic, D. 2019, ApJ, 876,85

Rigault, M., Aldering, G., Kowalski, M., et al. 2015, ApJ, 802, 20

Rigault, M., Brinnel, V., Aldering, G., et al. 2018, A\&A, submitted [arXiv:1806.03849]

Roman, M., Hardin, D., Betoule, M., et al. 2018, A\&A, 615, A68

Rusu, C. E., Fassnacht, C. D., Sluse, D., et al. 2017, MNRAS, 467, 4220

Rusu, C. E., Wong, K. C., Bonvin, V., et al. 2019a, MNRAS, submitted [arXiv:1905.09338]

Rusu, C. E., Berghea, C. T., Fassnacht, C. D., et al. 2019b, MNRAS, 486, 4987

Schmidt, B. P., Kirshner, R. P., Eastman, R. G., et al. 1994, ApJ, 432, 42

Schutz, B. F. 1986, Nature, 323, 310

Shajib, A. J., Treu, T., \& Agnello, A. 2018, MNRAS, 473, 210

Sluse, D., Sonnenfeld, A., Rumbaugh, N., et al. 2017, MNRAS, 470, 4838

Spiniello, C., Agnello, A., Napolitano, N. R., et al. 2018, MNRAS, 480, 1163

Sullivan, M., Conley, A., Howell, D. A., et al. 2010, MNRAS, 406, 782

Suyu, S. H., Marshall, P. J., Auger, M. W., et al. 2010, ApJ, 711, 201

Suyu, S. H., Treu, T., Hilbert, S., et al. 2014, ApJ, 788, L35

Suyu, S. H., Bonvin, V., Courbin, F., et al. 2017, MNRAS, 468, 2590

The LIGO Scientific Collaboration, the Virgo Collaboration, the $1 \mathrm{M} 2 \mathrm{H}$ Collaboration, et al. 2017, Nature, 551, 85

Tihhonova, O., Courbin, F., Harvey, D., et al. 2018, MNRAS, 477, 5657

Treu, T., \& Marshall, P. J. 2016, A\&ARv, 24, 11

Treu, T., Agnello, A., Baumer, M. A., et al. 2018, MNRAS, 481, 1041

Vattis, K., Koushiappas, S. M., \& Loeb, A. 2019, Phys. Rev. D, 99, 121302

Wojtak, R., \& Agnello, A. 2019, MNRAS, 486, 5046

Wong, K. C., Suyu, S. H., Auger, M. W., et al. 2017, MNRAS, 465, 4895 